\title{
]jfis
}

\section{Generalized Fuzzy Ideal Closed Sets on Fuzzy Topological Spaces in Sostak Sense}

Yasser M. Saber ${ }^{1,2}$ and Fahad Alsharari ${ }^{2}$

${ }^{1}$ Department of Mathematics, Faculty of Science, Al-Azhar University, Assiut Egypt

${ }^{2}$ Department of Mathematics, College of Science and Human Studies of Hotat Sudair, Majmaah University, Majmaah, Saudi Arabia
Received: Apr. 4, 2018

Revised : Aug. 21, 2018

Accepted: Sep. 18, 2018

Correspondence to: Yasser M. Saber (m.ah75@yahoo.com)

(CThe Korean Institute of Intelligent Systems

(c) This is an Open Access article distributed under the terms of the Creative Commons Attribution Non-Commercial License (http://creativecommons.org/licenses/ by-nc/3.0/] which permits unrestricted noncommercial use, distribution, and reproduction in any medium, provided the original work is properly cited.

\begin{abstract}
Recently, El-Naschie has shown that the notion of fuzzy topology may be relevant to quantum paretical physics in connection with string theory and E-infinity space time theory. In this paper, we define concept r-generalized fuzzy ideal closed sets with respect to an fuzzy ideal topological space in Sostak sense.We investigate some properties of them, we investigate the relationships between r-generalized fuzzy ideal closed sets with respect to an ideal and r-fuzzy separated
\end{abstract}

Keywords: r-generalized fuzzy closed sets, r-generalized fuzzy closed sets with respect to an fuzzy ideal topological space in Sostak sense, r-fuzzy separated

\section{Introduction}

Sostak [1], introduce the fundamental concept of fuzzy topological structure as an extension of both crisp topology and Chang's fuzzy topology [2], in the sense that not only the object were fuzzified, but also the axiomatics. Chattopdhyay et al. [3] and El Naschie [4] have redefined the similar concept. In [5], the author gave a similar definition namely "Smooth fuzzy topology". We must point out that; the concept of fuzzy topological spaces, has been a significant concept in string theory and E-infinity theory pertaining to quantum particular physics ever since El-Naschie [6-14]. After that several authors [15-17] have introduced the smooth definition and studied smooth fuzzy ideai topological spaces being unaware of Sostak works.

Throughout this paper, let $X$ be a nonempty set $I=[0,1]$ and $I_{0}=(0,1]$. For $\alpha \in$ $I, \bar{\alpha}(x)=\alpha$ for all $x \in X$. The family of all fuzzy sets on $X$ denoted by $I^{X}$. For two fuzzy sets we write $\lambda q \mu$ to mean that $\lambda$ is quasi-coincident (q-coincident, for short) with $\mu$, i.e, there exists at least one point $x \in X$ such that $\lambda(x)+\mu(x)>1$. Negation of such a statement is denoted as $\lambda \bar{q} \mu$.

Definition 1.1 ( [1] ). A mapping $\tau: I^{X} \rightarrow I$ is called a fuzzy topology on $X$ if it satisfies the following conditions:

(O1) $\tau(\overline{0})=\tau(\overline{1})=\overline{1}$.

(O2) $\tau\left(\bigvee_{i \in \Gamma} \mu_{i}\right) \geq \bigwedge_{i \in \Gamma} \tau\left(\mu_{i}\right)$, for any $\left\{\mu_{i}\right\}_{i \in \Gamma} \in I^{X}$.

(O3) $\tau\left(\mu_{1} \wedge \mu_{2}\right) \geq \tau\left(\mu_{1}\right) \wedge \tau\left(\mu_{2}\right)$, for any $\mu_{1}, \mu_{2} \in I^{X}$. 
The pair $(X, \tau)$ is called a fuzzy topological space (for short, fts).

Definition $1.2([18])$. Let $(X, \tau)$ be a fts, $\lambda, \mu \in I^{X}$ and $r \in I_{0}$.

1) A fuzzy set $\lambda$ is called r-generalized fuzzy closed (for short, r-gfc) if $C_{\tau}(\lambda, r) \leq \mu$ whenever $\lambda \leq \mu$ and $\tau(\mu) \geq r$.

2) A fuzzy set $\lambda$ is called r-generalized fuzzy open (for short, r-gfo) if $I_{\tau}(\lambda, r) \geq \mu$ whenever $\lambda \geq \mu$ and $\tau(\overline{1}-\mu) \geq r$.

Definition $1.3([1,5,15,16])$. A mapping $\mathbf{I}: I^{X} \rightarrow I$ is called fuzzy ideal on $\mathrm{X}$ iff:

1) $\mathbf{I}(\underline{0})=1, \mathbf{I}(\underline{1})=0$.

2) If $\lambda \leq \mu$, then $\mathbf{I}(\lambda) \geq \mathbf{I}(\mu)$, for each $\lambda, \mu \in I^{X}$.

3) For each $\lambda, \mu \in I^{X}, \quad \mathbf{I}(\lambda \vee \mu) \geq \mathbf{I}(\lambda) \wedge \mathbf{I}(\mu)$ [finite additivity].

Lemma 1.4. Let $(X, \tau, \mathcal{I})$ be a fits. The simplest fuzzy ideal on $X$ are $\mathcal{I}^{0}, \mathcal{I}^{1}: I^{X} \rightarrow I$ where

$$
\begin{aligned}
& \mathcal{I}^{1}(\lambda)= \begin{cases}1, & \text { if } \lambda=\underline{0}, \\
0, & \text { otherwise. }\end{cases} \\
& \mathcal{I}^{0}(\lambda)= \begin{cases}0, & \text { if } \lambda=\underline{1}, \\
1, & \text { otherwise. }\end{cases}
\end{aligned}
$$

If we take $\mathcal{I}=\mathcal{I}^{0}$, for each $\mathcal{A} \in I^{X}$ we have $\mathcal{A}_{r}^{*}=C_{\tau}(\mathcal{A}, r)$.

If we take $\mathcal{I}=\mathcal{I}^{1}$, for each $\mathcal{A} \in \Theta^{\prime}$ we have $\mathcal{A}_{r}^{*}=\underline{0}$, where, $\underline{1} \notin \Theta^{\prime}$ be a subset of $I^{X}$.

Definition 1.5 ( [19]). Let $(X, \tau, \mathbf{I})$ be a fuzzy ideal topological space. Let $\mu, \lambda \in I^{X}$, the r-fuzzy open local function $\mu_{r}^{*}$ of $\mu$ is the union of all fuzzy points $x_{t}$ such that if $\rho \in Q\left(x_{t}, r\right)$ and $\mathbf{I}(\lambda) \geq r$ then there is at least one $y \in X$ for which $\rho(y)+\mu(y)-1>\lambda(y)$.

Theorem 1.6 ( [4]). Let $(X, \tau)$ be a fts. Then for each $r \in$ $I_{0}, \lambda \in I^{X}$ we define an operator $C_{\tau}: I^{X} \times I_{0} \rightarrow I^{X}$ as follows:

$$
C_{\tau}(\lambda, r)=\bigwedge\left\{\mu \in I^{X}: \lambda \leq \mu, \tau(\overline{1}-\mu) \geq r\right\}
$$

For $\lambda, \mu \in I^{X}$ and $r, s \in I_{0}$, the operator $C_{\tau}$ satisfies the following conditions:

1) $C_{\tau}(\overline{0}, r)=\overline{0}$.
2) $\lambda \leq C_{\tau}(\lambda, r)$

3) $C_{\tau}(\lambda, r) \vee C_{\tau}(\mu, r)=C_{\tau}(\lambda \vee \mu, r)$.

4) $C_{\tau}(\lambda, r) \leq C_{\tau}(\lambda, s)$ if $r \leq s$.

5) $C_{\tau}\left(C_{\tau}(\lambda, r), r\right)=C_{\tau}(\lambda, r)$.

Theorem 1.7 ( $[20])$. Let $(X, \tau)$ be a fts. Then for each $r \in$ $I_{0}, \lambda \in I^{X}$ we define an operator $I_{\tau}: I^{X} \times I_{0} \rightarrow I^{X}$ as follows:

$$
I_{\tau}(\lambda, r)=\bigvee\left\{\mu \in I^{X}: \lambda \geq \mu, \tau(\mu) \geq r\right\}
$$

For $\lambda, \mu \in I^{X}$ and $r, s \in I_{0}$, the operator $I_{\tau}$ satisfies the following conditions:

1) $I_{\tau}(\overline{1}-\lambda, r)=\overline{1}-C_{\tau}(\lambda, r)$ and $C_{\tau}(\overline{1}-\lambda, r)=\overline{1}-$ $I_{\tau}(\lambda, r)$.

2) $I_{\tau}(\overline{1}, r)=\overline{1}$.

3) $\lambda \geq I_{\tau}(\lambda, r)$.

4) $I_{\tau}(\lambda, r) \wedge I_{\tau}(\mu, r)=I_{\tau}(\lambda \wedge \mu, r)$.

5) $I_{\tau}(\lambda, r) \leq I_{\tau}(\lambda, s)$ if $r \geq s$.

6) $I_{\tau}\left(I_{\tau}(\lambda, r), r\right)=I_{\tau}(\lambda, r)$.

\section{2. r-generalized Fuzzy Closed Sets with Respect to an Ideal}

Definition 2.1. Let $(X, \tau, \mathbf{I})$ be fuzzy ideal topological space, $\mu \in I^{X}$ and $r \in I_{0}$. A fuzzy set $\mu$ is called r-generalized fuzzy closed with respect to an ideal (briefly, r-gfIc) if $\mathbf{I}\left(C_{\tau}(\mu, r) \backslash\right.$ $\lambda) \geq r$, whenever $\mu \leq \lambda$ and $\tau(\lambda) \geq r$

Lemma 2.2. Every r-gfc set is r-gfIc.

Proof. Let $\mu \leq \lambda$ and $\tau(\lambda) \geq r$. Since $\mu$ is r-gfc set, then $C_{\tau}(\mu, r) \leq \lambda$, this implies that $C_{\tau}(\mu, r) \bar{q} \underline{1}-\lambda$, implies $C_{\tau}(\mu, r)(x)+(\underline{1}-\lambda)(x) \leq 1$, then $C_{\tau}(\mu, r)(x)-\lambda(x) \leq 0$. Thus, $\mathbf{I}\left(C_{\tau}(\mu, r) \backslash \lambda\right) \geq r$.

Example 2.3. The converse Lemma 2.2 is not true. Let $X=$ $\{a, b, c\}$ be a set and $\alpha, \beta, \gamma \in I^{X}$ are defined as follows:

$$
\begin{aligned}
& \alpha(a)=0.2, \alpha(b)=0.4 ; \alpha(c)=0.7 \\
& \beta(a)=0.7, \beta(b)=0.6 ; \beta(c)=0.8 \\
& \gamma(a)=0.6 ; \gamma(b)=0.4, \gamma(c)=0.7 .
\end{aligned}
$$


We define fuzzy topology and fuzzy ideal $\tau, \mathbf{I}: I^{X} \rightarrow I$ as follows:

$$
\begin{aligned}
& \tau(\lambda)= \begin{cases}1, & \text { if } \nu=\underline{1}, \underline{0}, \\
\frac{1}{2}, & \text { if } \nu=\alpha, \\
\frac{1}{2}, & \text { if } \nu=\beta, \\
0, & \text { otherwise. }\end{cases} \\
& \mathbb{I}(\lambda)= \begin{cases}1, & \text { if } \nu=\underline{0}, \\
\frac{1}{2}, & \text { if } \nu=\underline{0.3}, \\
\frac{1}{2}, & \text { if } \underline{0}<\nu<\underline{0.3}, \\
0, & \text { otherwise, }\end{cases}
\end{aligned}
$$

For $r=\frac{1}{3}, \underline{1}-\gamma$ is r-gfIc set, where

$$
\begin{aligned}
& \underline{1}-\gamma \leq \beta, \quad \tau(\beta) \geq \frac{1}{3} \\
& C_{\tau}\left(\underline{1}-\gamma, \frac{1}{3}\right)=\underline{1}-\alpha \backslash \beta=a_{0.3} .
\end{aligned}
$$

Therefore, $\mathbf{I}\left(\left(C_{\tau}\left(\underline{1}-\gamma, \frac{1}{3}\right) \backslash \alpha\right), \frac{1}{3}\right) \geq \frac{1}{3}$.

But $\underline{1}-\gamma$ is not $r$-gfc set because

$$
\underline{1}-\gamma \leq \beta, \quad \tau(\beta) \geq \frac{1}{3}, \quad\left(C_{\tau}\left(\underline{1}-\gamma, \frac{1}{3}\right)=\underline{1}-\alpha\right) \geq \beta .
$$

Theorem 2.4. Let $(X, \tau, \mathbf{I})$ be an fuzzy ideal topological space, $\mu, \lambda \in I^{X}$ and $r \in I_{0}$. If $\mu$ and $\lambda$ are r-gfIc sets, then $\mu \vee \lambda$ is r-gfIc.

Proof. Suppose $\mu$ and $\lambda$ are r-gfIc sets. If $\mu \vee \lambda \leq \rho$ and $\tau(\rho) \geq$ $r$, then $\mu \leq \rho$ and $\lambda \leq \rho$. By assumption, $\mathbf{I}\left(C_{\tau}(\mu, r) \backslash \rho\right) \geq r$ and $\mathbf{I}\left(C_{\tau}(\lambda, r) \backslash \rho\right) \geq r$ and hence

$$
\mathbf{I}\left(C_{\tau}(\mu \vee \lambda, r) \backslash \rho=C_{\tau}(\mu, r) \backslash \rho \vee C_{\tau}(\lambda, r) \backslash \rho\right) \geq r
$$

Therefore, $\mu \vee \lambda$ is r-gfIc.

Remark 2.5. The intersection of two r-gfIc sets need not be an r-gfIc set as shown by the following example.

Example 2.6. Let $X=\{a, b, c\}$ be a set and $\alpha, \beta, \gamma \in I^{X}$ are defined as follows:

$$
\begin{aligned}
& \alpha(a)=0.8, \alpha(b)=0.4 ; \alpha(c)=0.7 \\
& \beta(a)=0.6, \beta(b)=0.5 ; \beta(c)=0.8 \\
& \gamma(a)=0.6 ; \gamma(b)=0.4, \gamma(c)=0.7 .
\end{aligned}
$$

We define fuzzy topology and fuzzy ideal $\tau, \mathbf{I}: I^{X} \rightarrow I$ as follows:

$$
\begin{aligned}
& \tau(\lambda)= \begin{cases}1, & \text { if } \nu=\underline{1}, \underline{0}, \\
\frac{1}{2}, & \text { if } \nu=\gamma \\
0, & \text { otherwise, }\end{cases} \\
& \mathbb{I}(\lambda)= \begin{cases}1, & \text { if } \nu=\underline{0}, \\
\frac{1}{2}, & \text { if } \nu=\underline{0.3}, \\
\frac{1}{2}, & \text { if } \underline{0}<\nu<\underline{0} .3, \\
0, & \text { otherwise. }\end{cases}
\end{aligned}
$$

For $r=\frac{1}{3}, \beta$ and $\gamma$ is r-gfIc set. But $\beta \wedge \gamma=\gamma$ is not r-gfIc set because $\gamma \leq \gamma, \quad \tau(\gamma) \geq r, C_{\tau}\left(\gamma, \frac{1}{3}\right)=\underline{1} \backslash \gamma=\underline{1}$. Therefore $\mathbf{I}\left(\left(C_{\tau}\left(\gamma, \frac{1}{3}\right) \backslash \gamma\right), \frac{1}{3}\right)<\frac{1}{3}$.

Theorem 2.7. Let $(X, \tau, \mathbf{I})$ be an fuzzy ideal topological space, $\mu, \lambda \in I^{X}$ and $r \in I_{0}$. If $\mu$ is r-gfIc set and $\mu \leq \lambda \leq C_{\tau}(\mu, r)$, then $\lambda$ are r-gfIc.

Proof. Let $\mu$ is r-gfIc set and $\mu \leq \lambda \leq C_{\tau}(\mu, r)$. Suppose $\lambda \leq \rho$ and $\tau(\rho) \geq r$. Then $\mu \leq \rho$. Since $\mu$ is r-gfIc, we have $\mathbf{I}\left(C_{\tau}(\mu, r) \backslash \rho\right) \geq r$. Now $\lambda \leq C_{\tau}(\mu, r)$ implies that

$$
C_{\tau}(\lambda, r) \backslash \rho \leq C_{\tau}(\mu, r) \backslash \rho
$$

and hence, $\mathbf{I}\left(C_{\tau}(\lambda, r) \backslash \rho\right) \geq r$. Therefore, $\lambda$ is r-gfIc set.

Definition 2.8. Let $(X, \tau, \mathbf{I})$ be fuzzy ideal topological space, $\mu \in I^{X}$ and $r \in I_{0}$. A fuzzy set $\mu$ is called r-fuzzy generalized open with respect to an ideal I (briefly, r-gfIo) if $\underline{1}-\mu$ is r-gfIc set.

Theorem 2.9. Let $(X, \tau, \mathbf{I})$ be an fuzzy ideal topological space, $\mu, \lambda, \rho \in I^{X}$ and $r \in I_{0}$. If $\mu$ is r-gfIo sets if and only if $\lambda \backslash \rho \leq I_{\tau}(\mu, r)$ for some $\mathbf{I}(\rho) \geq r$, whenever $\lambda \leq \mu$ and $\tau(\underline{1}-\lambda) \geq r$.

Proof. Suppose that $\mu$ is r-gfIo sets. Suppose $\lambda \leq \mu$ and $\tau(\underline{1}-\lambda) \geq r$. We have $\underline{1}-\lambda \geq \underline{1}-\mu$. By assumption,

$$
C_{\tau}(\underline{1}-\mu, r) \leq \underline{1}-\lambda \vee \rho .
$$

For some $\mathbf{I}(\rho) \geq r$. This implies

$$
\underline{1}-((\underline{1}-\lambda) \vee \rho) \leq \underline{1}-C_{\tau}(\underline{1}-\mu),
$$

and hence, $\lambda \backslash \rho \leq I_{\tau}(\mu, r)$. 
Conversely, assume that $\lambda \leq \mu$ and $\tau(\underline{1}-\lambda) \geq r$ imply $\lambda \backslash \rho \leq I_{\tau}(\mu, r)$ for some $\mathbf{I}(\rho) \geq r$. Consider $\tau(\omega) \geq r$ such that $\underline{1}-\mu \leq \omega$. Then $\underline{1}-\omega \leq \mu$. By assumption,

$$
\underline{1}-\omega \backslash \rho \leq I_{\tau}(\mu, r)=\underline{1}-C_{\tau}(\underline{1}-\mu, r)
$$

For some $\mathbf{I}(\rho) \geq r$. This gives that

$$
\underline{1}-(\omega \vee \rho) \leq \underline{1}-C_{\tau}(\underline{1}-\mu, r) .
$$

Therefore, $C_{\tau}(\underline{1}-\mu, r) \leq \omega \vee \rho$, for some $\mathbf{I}(\rho) \geq r$. This show that $\mathbf{I}\left(C_{\tau}(\underline{1}-\mu, r) \backslash \omega\right) \geq r$. Hence, $\underline{1}-\mu$ is r-gfIc set.

Recall that the sets $\mu$ and $\lambda$ are fuzzy separated if $C_{\tau}(\mu, r) \bar{q} \lambda$ and $\mu \bar{q} C_{\tau}(\lambda, r)$.

Theorem 2.10. Let $(X, \tau, \mathbf{I})$ be an fuzzy ideal topological space, $\mu, \lambda, \in I^{X}$ and $r \in I_{0}$. If $\mu$ and $\lambda$ are fuzzy separated and r-gfIo sets, then $\mu \vee \lambda$ is r-gfIo.

Proof. Suppose $\mu$ and $\lambda$ are fuzzy separated and r-gflo sets and $\rho \leq \mu \vee \lambda$, and $\tau(\underline{1}-\rho) \geq r$. Then $\rho \wedge C_{\tau}(\mu, r) \leq \mu$ and $\rho \wedge C_{\tau}(\lambda, r) \leq \lambda$. By assumption,

$$
\begin{aligned}
& \rho \wedge C_{\tau}(\mu, r) \backslash \nu_{1} \leq I_{\tau}(\mu, r), \\
& \rho \wedge C_{\tau}(\lambda, r) \backslash \nu_{2} \leq I_{\tau}(\lambda, r),
\end{aligned}
$$

for some $\mathbf{I}\left(\left(\nu_{1}, \nu_{2}\right), r\right) \geq r$. This means

$$
\mathbf{I}\left(\rho \wedge C_{\tau}(\mu, r) \backslash I_{\tau}(\mu, r), r\right) \geq r
$$

and

$$
\mathbf{I}\left(\rho \wedge C_{\tau}(\lambda, r) \backslash I_{\tau}(\lambda, r), r\right) \geq r .
$$

Thus,

$$
\begin{aligned}
& \mathbf{I}\left(\left(\rho \wedge C_{\tau}(\mu, r) \backslash I_{\tau}(\mu, r)\right) \vee\left(\rho \wedge C_{\tau}(\lambda, r)\right) \backslash I_{\tau}(\lambda, r), r\right) \\
& \quad \geq r .
\end{aligned}
$$

Therefore,

$$
\begin{aligned}
& \mathbf{I}\left(\rho \wedge\left(C_{\tau}(\mu, r) \vee C_{\tau}(\lambda, r)\right) \backslash\left(I_{\tau}(\mu, r) \vee I_{\tau}(\lambda, r)\right), r\right) \\
& \quad \geq r .
\end{aligned}
$$

But

$$
\rho=\rho \wedge(\mu \vee \lambda) \leq \rho \wedge\left(C_{\tau}(\mu \vee \lambda, r)\right),
$$

and we have

$$
\mathbf{I}\left(\rho \backslash I_{\tau}(\mu \vee \lambda, r) \leq\left(\rho \wedge C_{\tau}(\mu \vee \lambda, r)\right) \backslash I_{\tau}(\mu \vee \lambda, r)\right.
$$

$$
\left.\leq\left(\rho \wedge C_{\tau}(\mu \vee \lambda, r)\right) \backslash\left(I_{\tau}(\mu, r) \vee I_{\tau}(\lambda, r)\right)\right) \geq r .
$$

Hence, $\rho \backslash \nu \leq \operatorname{Int}_{\tau}(\mu \vee \lambda, r)$ for some $\mathbf{I}(\nu) \geq r$. This proves that $\mu \vee \lambda$ is r-gfIo.

Corollary 2.11. Let $(X, \tau, \mathbf{I})$ be an fuzzy ideal topological space, $\mu, \lambda, \in I^{X}$ and $r \in I_{0}$. If $\mu$ and $\lambda$ are r-gflo sets, $\underline{1}-\mu$ and $\underline{1}-\lambda$ are fuzzy separated. Then $\mu \wedge \lambda$ is r-gfIc.

Proof. Obvious.

Corollary 2.12. Let $(X, \tau, \mathbf{I})$ be an fuzzy ideal topological space, $\mu, \lambda, \in I^{X}$ and $r \in I_{0}$. If $\mu$ and $\lambda$ are r-gfIo sets, then $\mu \wedge \lambda$ is r-gfIo.

Proof. Obvious.

Theorem 2.13. Let $(X, \tau, \mathbf{I})$ be an fuzzy ideal topological space, $\mu, \lambda, \in I^{X}$ and $r \in I_{0}$. If and $\mu \leq \lambda$, and $\mu$ r-gfIo relative to $\lambda$ and $\lambda$ is r-gfIo relative to $X$, then $\mu$ r-gfIo relative to $X$.

Proof. Suppose that $\mu \leq \lambda, \mu$ is r-gfIo relative to $\lambda$ and $\lambda$ is r-gflo relative to $X$. Let $\rho \leq \mu$ and $\tau(\underline{1}-\rho) \geq r$. Since $\mu$ is r-gflo relative to $\lambda$. By Theorem 2.9, $\rho \backslash \nu_{1} \leq I_{\lambda}(\mu, r)$ for some $\mathbf{I}\left(\nu_{1}\right) \geq r$. This implies that there exists $\tau\left(\omega_{1}\right) \geq r$ such that

$$
\rho \backslash \nu_{1} \leq \omega_{1} \wedge \lambda \leq \mu,
$$

for some $\mathbf{I}\left(\nu_{1}\right) \geq r$. Let $\rho \leq \lambda$ and $\tau(\underline{1}-\rho) \geq r$. Since $\lambda$ is r-gfIo, we have

$$
\rho \backslash \nu_{2} \leq \operatorname{Int}_{\tau}(\lambda, r)
$$

for some $\mathbf{I}\left(\nu_{2}\right) \geq r$. This implies that there exists $\tau\left(\omega_{2}\right) \geq r$ such that

$$
\rho \backslash \nu_{2} \leq \omega_{2} \leq \lambda,
$$

for some $\mathbf{I}\left(\nu_{2}\right) \geq r$. Now

$\rho \backslash\left(\nu_{1} \vee \nu_{2}\right)=\left(\rho \backslash \nu_{1}\right) \wedge\left(\rho \backslash \nu_{2}\right) \leq \omega_{1} \wedge \omega_{2} \leq \omega_{1} \wedge \lambda \leq \mu$.

This implies that $\rho \backslash\left(\nu_{1} \vee \nu_{2}\right) \leq I_{\lambda}(\mu, r)$ for some $\mathbf{I}\left(\nu_{1} \vee \nu_{2}\right) \geq$ $r$. Thus, $\mu$ r-gflo relative to $X$.

\section{Conflict of Interest}

No potential conflict of interest relevant to this article was reported. 


\section{Acknowledgements}

The authors are grateful for the support by Faculty of Science and Humanities, Majmaah University.

\section{References}

[1] A. P. Sostak, “On a fuzzy topological structure," in Proceedings of the 13th Winter School on Abstract Analysis (Circolo Matematico di Palermo), Palermo, Italy, pp. 89$103,1985$.

[2] C. L. Chang, "Fuzzy topological spaces," Journal of Mathematical Analysis and Applications, vol. 24, no. 1, pp. 182-190, 1968.

[3] K. C. Chattopadhyay, R. N. Hazra, and S. K. Samanta, "Gradation of openness: fuzzy topology," Fuzzy Sets and Systems, vol. 49, no. 2, pp. 237-242, 1992. https://doi.org/ 10.1016/0165-0114(92)90329-3

[4] M. S. El Naschie, "Advanced prerequisite for E-infinity theory," Chaos, Solitons \& Fractals, vol. 30, no. 3, pp. 636641, 2006. https://doi.org/10.1016/j.chaos.2006.04.044

[5] A. A. Ramadan. ?Smooth topological spaces," Fuzzy Sets and Systems, vol. 48, no. 3, pp. 371-375, 1992. https: //doi.org/10.1016/0165-0114(92)90352-5

[6] M. S. El Naschie, "A review of E-infinity theory and the mass spectrum of high energy particle physics," Chaos, Solitons \& Fractals, vol. 19, no. 1, pp. 209-236, 2004. https://doi.org/10.1016/S0960-0779(03)00278-9

[7] M. S. El Naschie, "Elementary prerequisite for E-infinity (recommended background readings in nonlinear dynamics, geometry and topology)," Chaos, Solitons \& Fractals, vol. 30, no. 3, pp. 579-606, 2006. https://doi.org/10.1016/ j.chaos.2006.03.030

[8] M. S. El Naschie, “On a fuzzy Kahler-like manifold which is consistent with the two slit experiment," International Journal of Nonlinear Sciences and Numerical Simulation, vol. 6, no. 2, pp. 95-98, 2005. https://doi.org/10.1515/ IJNSNS.2005.6.2.95

[9] M. S. El Naschie, "On the uncertainty of Cantorian geometry and the two-slit experiment," Chaos, Solitons \& Fractals, vol. 30, no. 3, pp. 517-529, 1998. https: //doi.org/10.1016/S0960-0779(97)00150-1
[10] M. S. El Naschie, "On the unification of heterotic strings, M theory and E $(\infty)$ theory," Chaos, Solitons \& Fractals, vol. 11, no. 14, pp. 2397-2408, 2000. https://doi.org/10. 1016/S0960-0779(00)00108-9

[11] M. S. El Naschie, "Quantum gravity from descriptive set theory," Chaos, Solitons \& Fractals, vol. 19, no. 5, pp. 1339-1344, 2004. https://doi.org/10.1016/j.chaos.2003.08. 009

[12] M. S. El Naschie, "Quantum gravity, Clifford algebras, fuzzy set theory and the fundamental constants of nature,' Chaos, Solitons \& Fractals, vol. 20, no. 3, pp. 437-450, 2004. https://doi.org/10.1016/j.chaos.2003.09.029

[13] M. S. El Naschie, "The simplictic vacuum, exotic quasiparticles and gravitational instanton," Chaos, Solitons \& Fractals, vol. 22, no. 1, pp. 1-11, 2004.https://doi.org/10. 1016/j.chaos.2004.01.015

[14] M. S. El Naschie, "Topics in the mathematical physics of E-infinity theory," Chaos, Solitons \& Fractals, vol. 30, no. 3, pp. 656-663, 2006. https://doi.org/10.1016/j.chaos 2006.04 .043

[15] A. M. Zahran, S. A. Abd El-Baki, and Y. M. Saber, "Decomposition of fuzzy ideal continuity via fuzzy idealization," International Journal of Fuzzy Logic and Intelligent Systems, vol. 9, no. 2, pp. 83-93, 2009. https: //doi.org/10.5391/IJFIS.2009.9.2.083

[16] A. M. Zahran, S. E. Abbas, S. A. Abd El-baki, and Y M. Saber, "Decomposition of fuzzy continuity and fuzzy ideal continuity via fuzzy idealization," Chaos, Solitons \& Fractals, vol. 42, no. 5, pp. 3064-3077, 2009. https: //doi.org/10.1016/j.chaos.2009.04.010

[17] S. A. Abd El-Baki and Y. M. Saber, "Fuzzy extremally disconnected ideal topological spaces,' International Journal of Fuzzy Logic and Intelligent Systems, vol. 10, no. 1, pp $1-6,2010$.

[18] Y. C. Kim and J. M. Ko, "r-generalized fuzzy closed sets," Journal of Fuzzy Mathematics, vol. 12, no. 1, pp. 7-21, 2004.

[19] Y. M. Saber and M. A. Abdel-Sattar, "Ideals on fuzzy topological spaces," Applied Mathematical Sciences, vol. 8, no. 34, pp. 1667-1691, 2014. https://doi.org/10.12988/ ams.2014.33194 
[20] A. A. Ramadan, S. E. Abbas, and Y. C. Kim, "Fuzzy irresolute functions in smooth fuzzy topological space," Journal of Fuzzy Mathematics, vol. 9, no. 4, pp. 865-877, 2001.

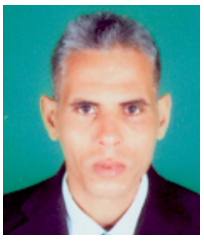

Yasser M. Saber received B.Sc., M.Sc., and Ph.D., in Faculty of Science, Al-Azhar University, Egypt, in 1998, 2006, and 2010, respectively. From 1998 to 2006, he has worked as a Demonstrator in Faculty of Science, Al-Azhar University. From 2006 to 2010, he has worked as Assistant Lecturer in Faculty of Science, Al-Azhar University, Egypt. From 2011 until now he has been worked as an Assistant Professor in Faculty of Science, Al-Azhar University, Egypt. From 2014 until now, he has been worked as an Assistant Professor in College of Science and Human studies of Hotat Sudair, Majmaah
University, Majmaah. His research interests general topology, fuzzy topology and Applications of general and fuzzy topology. E-mail:m.ah75@yahoo.com

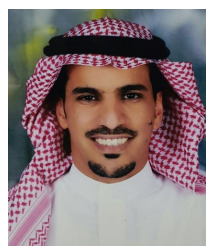

Fahad Alsharari I received his B.Sc. in mathematics in 2006 from Jouf University and his M.Sc. and Ph.D. from Faculty of Science and Technology, National University of Malaysia, Malaysia in 2011 and 2016, respectively. From 2017 until now, he has been worked as an Assistant Professor in College of Science and Human studies of Hotat Sudair, Majmaah University, Majmaah. His research interests general topology, fuzzy topology and Applications of general and fuzzy topology.

E-mail: f.alsharari@mu.edu.sa. 